\title{
Secondary radial neuropathy after closed intramedullary nailing of humeral shaft fractures. Results over a 10-year period
}

\author{
Anton UlsTRUP \\ From the Department of Orthopaedic Surgery, Holbcek Hospital, Smedelundsgade 60, 4300 Holbcek, Denmark
}

Background. Retrospective study to examine secondary radial nerve palsy after humeral shaft fixation with closed locked intramedullary nailing. Materials and methods. Patients were identified from the hospitals' registration systems for humeral shaft fractures, nerve lesions, plating, nailing and external fixation during a 10-year period from January 2007 to December 2016. All radial nerve lesions were registered and followed-up in patient files. Results. 89 patients with locked intramedullary nailing were available for an outpatient follow-up. Mean age was 67 years at the time of injury. 72 fractures were non-pathological. Of these, 31 were nonunions. 28, 61 and zero were identified in the proximal, middle and distal thirds of the humeral shaft respectively. 76 procedures were closed and 13 were with open reduction. Six radial nerves had nerve exploration. Eight patients developed immediate postoperative radial nerve palsies. Of these, six developed after closed surgery, two after nerve exploration. Of seven available patients with a radial nerve palsy, six of these remitted. Two patients were later surgically explored. One patient out of 89 sustained a verifiable permanent radial nerve paralysis. Conclusions. In this study, the risk of a radial nerve palsy was 7.9 $\%$ with closed locked intramedullary nailing. This study suggests that exploration of the radial nerve is not necessary routinely in order to prevent radial nerve lesions when performing closed intramedullary nailing for humeral shaft fractures in adults with a preoperative normal radial nerve function.

Level of Evidence : Level IV.
Keywords : Locked intramedullary nailing ; radial nerve palsy ; humeral shaft fracture ; nerve exploration.

\section{INTRODUCTION}

Intramedullary nailing of humeral shaft fractures (HSFs) using long nails is a classical procedure in a trauma or shoulder and elbow department. It is often performed in adults with locking screws and is recommended for simple as well as complex HSFs. Nails are also utilized prophylactically for certain pathological humeral bones to prevent fracture (1). Other surgical methods of fixation of humeral shaft fractures in adults include plating and external fixation (2-5).

In studies of patients with radial nerve palsy in relation to HSFs (RHSFs), a pooled incidence of approximately $12 \%$ is found, increased with highenergy trauma, with open lesions and possibly higher with plating than with intramedullary nailing.

Dr. Anton Ulstrup, M.D., Fellow of The European Board of Orthopaedics and Traumatology (FEBOT).

Department of Orthopaedic Surgery, Holboek Hospital, Smedelundsgade 60, 4300 Holbaek, Denmark.

Correspondence: Dr. Anton Ulstrup, Enighedsvej 3, 2920

Charlottenlund, Greater Copenhagen, Denmark. Phone : +4540155561.

Email : antonulstrup@gmail.com

- 2021, Acta Orthopædica Belgica. 
In some studies, the incidence is a little higher with mid-shaft or mid to distal-shaft fractures, and with transverse fractures and spiral fractures compared to oblique and comminuted fractures (3-4,8-9,13-14,17).

In the literature, it is generally recommended to identify the radial nerve when plating shaft fractures and there are a number of studies on plating associated with radial neuropathy (3-4,510). Considering fixation of HSFs with a long intramedullary nail, there are far fewer publications in English addressing that complication. Specifically, when nailing is performed as a closed procedure with no intraoperative identification of the radial nerve, it is less clear whether secondary radial nerve palsies are more or less prevalent compared to plating. A radial nerve palsy during closed nailing may be due to manipulation of the fracture, during intramedullary drilling or distal locking of the nail with screws (11-13).

Thus, a satisfying functional result in the treatment of adult HSFs with locked intramedullary nailing entails a preserved function of the radial nerve (14). The rate of radial nerve injury when introducing a nail into a reduced HSF with and without surgical exploration of the nerve and fracture site was investigated. In the author's local region with about 800.000 inhabitants, there are five orthopaedic departments that treat adult HSFs with any of plating, nailing or external fixation. In this retrospective study, outcome is reported and assessed specifically pertaining to the incidence of radial nerve palsy after the use of long locked intramedullary nailing for adult HSFs in five departments over a 10-year period. The results are compared to the literature.

\section{MATERIALS AND METHODS}

A search for patients from the five orthopaedic departments was conducted. Patients were identified from the hospitals' registration systems using operation and diagnosis codes for humeral shaft fractures, nerve lesions, plating, nailing and external fixation during a 10-year period from January 2007 to December 2016. The coding search was thus relatively extensive in order to increase the likelihood that all relevant fractures were included.
Acute fractures, delayed fractures and nonunions with nailing were included in the study. Only patients 18 years and older at the time of operation were included.

In this study, the S\&N Trigen intramedullary nailing system or the Stryker T2 humeral nail was used for internal fixation. At least two proximal locking screws and at least one distal screw were used in each operation. Indications were certain unstable fractures, nonunions and pathological, displaced fractures. Pathological fractures were all confirmed from histopathological analysis from bone biopsies. All included patients had intact radial nerve motor function prior to surgery.

The surgical procedures were performed according to the relevant manufacturer's recommendations and to accepted minimally invasive techniques published in the literature (16). 85 patients were referred to rehabilitation supervised by local municipal physiotherapists outside the hospital. With at least one follow-up visit for the patients, clinical results were described in the hospital file. Patients had at least two x-rays taken of the injured humerus including antero-posterior and lateral projections preoperatively and additionally, at least once during a follow-up visit. The relevant data on the eight patients with postoperative radial nerve palsies is presented in Table I.

Technically, for a closed procedure, a small anterolateral incision was made over the deltoid region and all distal locking was made through a small anterior incision distally. A posterior or an anterolateral incision over the fracture was performed in 13 cases in order to facilitate the introduction of the intramedullary guide wire and nail into the distal major fracture segment when closed attempts at this had failed. In only six cases was the radial nerve specifically identified and dissected.

Results were obtained by retrospectively reviewing all patient files as a cohort concerning admitted patients with an HSF within the 10-year period. All relevant radiographs were evaluated to verify the described implant and to classify the fractures according to the AO-OTA classification (15). Equal thirds of the shaft were measured on an antero-posterior radiograph and the third of the 


\begin{tabular}{|c|c|c|c|c|c|c|c|c|}
\hline 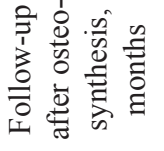 & 으 & $\infty$ & $r$ & - & $\dot{P}$ & $\infty$ & m & 0 \\
\hline 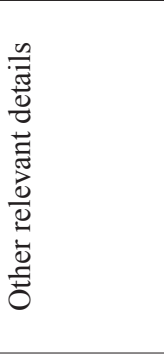 & 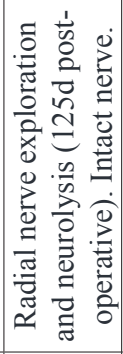 & & & & 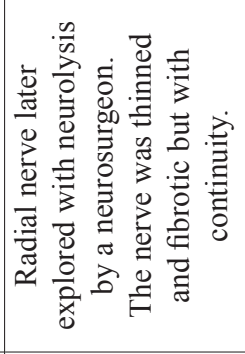 & 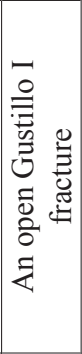 & & 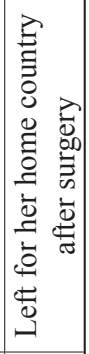 \\
\hline 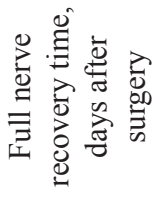 & $\widehat{\widehat{N}}$ & in & $\underset{n}{\infty}$ & $\stackrel{m}{m}$ & $\begin{array}{l}2 \\
0 \\
0 \\
0 \\
0 \\
0 \\
0 \\
0 \\
Z\end{array}$ & $\stackrel{\Delta}{\vec{N}}$ & $\hat{\alpha}$ & 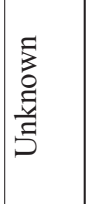 \\
\hline 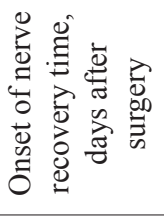 & 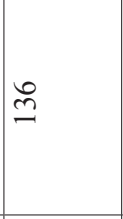 & 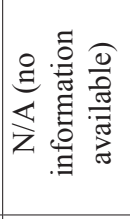 & $i n$ & 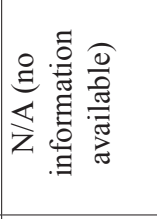 & 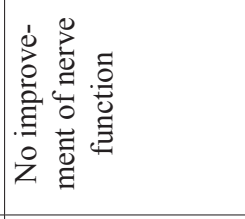 & $\underset{\nearrow}{\searrow}$ & $\bar{n}$ & 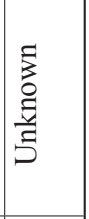 \\
\hline 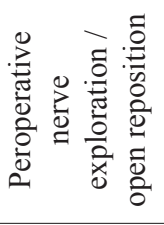 & 艺 & 苛 & 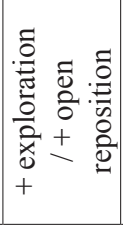 & $\ddot{\check{z}}$ & 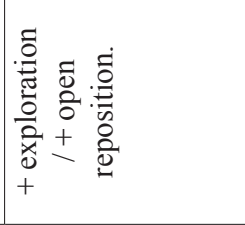 & 苂 & 苛 & 芩 \\
\hline 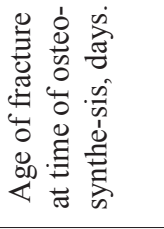 & $\stackrel{\sim}{\sim}$ & $\hat{n}$ & $\frac{n}{m}$ & in & $\stackrel{ \pm}{\sim}$ & - & in & $\curvearrowright$ \\
\hline 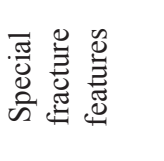 & & & 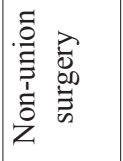 & 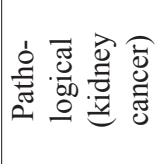 & 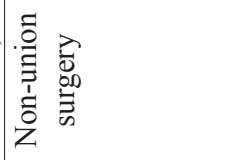 & & & \\
\hline 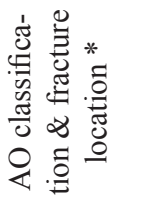 & 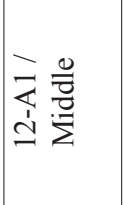 & 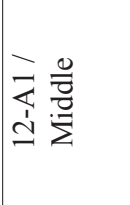 & 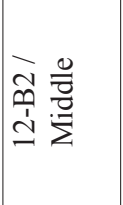 & 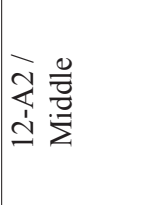 & 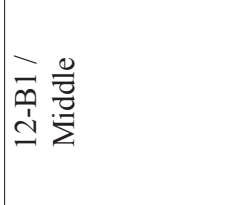 & 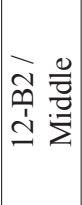 & 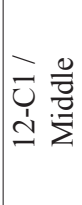 & 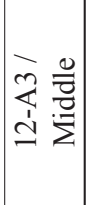 \\
\hline 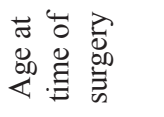 & $\stackrel{\infty}{+}$ & $\stackrel{\infty}{\sim}$ & $\mathbb{N}$ & $\mathbb{J}$ & $\bar{n}$ & $\infty$ & $\stackrel{0}{+}$ & $\tilde{\sigma}$ \\
\hline \multirow[t]{2}{*}{$\begin{array}{l}\overrightarrow{\bar{\theta}} \\
\overline{\bar{D}} \\
0\end{array}$} & 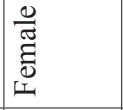 & 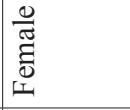 & 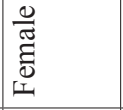 & 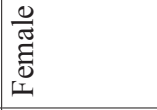 & 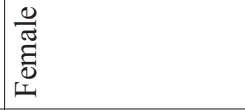 & $\frac{0}{\sum^{\pi}}$ & 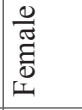 & 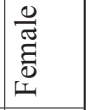 \\
\hline & - & $\sim$ & $m$ & $\nabla$ & in & 0 & $r$ & $\infty$ \\
\hline
\end{tabular}


humeral shaft, which the main part of the fracture belonged to, was identified.

\section{RESULTS}

89 patients were operated on with at least one clinical and radiographical outpatient review to evaluate the effect of the operation. Eight developed an immediate postoperative radial nerve palsy with complete motor loss $(9.0 \%)$. In five of the cases, the S\&N Trigen nailing system was used, and in three cases, the Stryker T2 humeral nail was used. There were 43 men and 46 women. Mean age was 67 years (range 18 to 92 ) at the time of injury. According to the AO classification, the group included 51 type A fractures, 15 type B fractures, and 23 type C fractures. 72 fractures were non-pathological, 17 were pathological and 31 were nonunions or delayed unions. 28, 61 and zero fractures were identified in the proximal, middle and distal thirds of the humeral shaft respectively. Six radial nerves were surgically explored at the time of nailing in the series. The mean follow-up period was 5.0 months (range one to 40).

\section{COMPLICATIONS}

After closed surgery six postoperative radial nerve palsies developed in 76 patients, and two developed after open surgery performed on two females whose fractures where operated on due to nonunion of AO-OTA type B mid-shaft fractures 214 days and 315 days old at the time of surgery, respectively. The other six cases had fracture ages ranging from one day to 53 days in five females and one male. Two secondary nerve explorations were performed. The nerves were found to be in continuity with no identifiable tethering, compression or entrapment. Neurolysis was done. Of the seven cases available for outpatient follow-up, six nerve palsies remitted. No other serious neurological or vascular complications were reported. Thus, there was a rate of $7.9 \%(6 / 76)$ for closed nailing and a rate of $15.4 \%(2 / 13)$ for nailing involving open surgery in the fracture area.

Because of the small sample size, the distributions of data cannot be regarded to be normal. Also, there were only eight patients with a secondary radial nerve palsy which is too small a sample size for a statistically viable comparative study. The study was under-powered. Therefore, the results are reported in a descriptive way.

\section{DISCUSSION}

Nerve exploration is recommended with open internal fixation by plating and with open juxtanervous fractures. This is to avoid placing a plate directly on the nerve and because there is a reported preponderance of visible nerve lesions with open fractures, probably because these fractures are due to high-energy trauma, where tissue is more heavily damaged than with low-energy trauma (3$4,7-10,13-14,18)$. In any case, there is a 7-32\% chance of sustaining an iatrogenic, secondary radial nerve palsy when performing open plating $(2-3,5,10-11,17)$ with possible risk variations related to the operative approach chosen (4).

Intramedullary nailing can be an excellent choice for internal fixation of certain unstable fracture types of humeral shaft fracture. In many cases, it can be performed in a minimally invasive fashion which has potential benefits for postoperative rehabilitation. In the literature, there are some studies that support abstaining from exploration with an RHSF after closed intramedullary nailing. This is because those studies find it rare to identify visible radial nerve lesions for secondary radial nerve palsy after closed procedures (2-3,7-13,17-18).

There was a rate of a radial nerve palsy of $7.9 \%$ (6/76) for closed nailing. This rate seems to be less compared to studies with plate internal fixation. The rate of a permanent nerve palsy $(1 / 88=1.1 \%)$ is probably what can be expected from a group of eight postoperative palsies. Pertaining to the 13 open surgery procedures, nine of which were for nonunion surgery, the associated rate of a permanent palsy is $7.7 \%$. This seems to be similar to or higher than results from other studies. The limitations of this study are its relatively short follow-up period for patients with a postoperative radial nerve lesion. Also, there are no equal groups of patients with or without nerve exploration to compare to each other. 
In about $90 \%$ of cases of RHSFs, including all fracture types and treatment modalities, radial nerve palsy remits. Of the cases that remit spontaneously, the start of the remission time is on average 7.3 weeks (range two weeks to 6.6 months), while the time to full remission is on average 6.1 months (range 3.4 months to 12 months) $(8-9,14)$. With RHSFs in general, some studies recommend observation for a period of time, that on average should be between three and six months. Based on a systematic review, Shao et al. (8) concluded that an expectant strategy to a closed RHSF was advisable and described a treatment algorithm (19). In this study, full remission in six patients available for follow-up was registered after a mean time of 127 days (33-227) which is shorter than the mean spontaneous recovery time in the literature (8).

When radial nerves are surgically explored, a risk of nerve lesion of approximately $10 \%$ is described, solely due to exploration $(3,5-6,11)$. If radial nerves in all cases of surgically treated HSFs were explored routinely, the implication would therefore be that a very large number of patients would be unnecessarily operated on, risking serious morbidities in so doing. It is also important to note that there is no difference in the rate for a radial nerve palsy to remit with either initial observation or late exploration compared to early exploration (8). This supports an expectant, conservative approach when closed surgery has been performed. A small minority of patients with an associated radial nerve palsy experience no satisfactory remission and for those cases, various techniques can be applied using specialized tendon transfers or nerve surgery procedures $(4,8-9)$.

\section{CONCLUSIONS}

Radial nerve palsy is a known complication for humeral shaft fractures and to surgical treatment thereof, including closed intramedullary nailing. The associated prognosis for spontaneous remission is good. As in some other studies on radial nerve lesions for open reduction and internal fixation with plating, this study had a group of patients with a limited rate of radial nerve lesions. A risk association was lower with closed nailing without nerve exploration compared to the cases with open surgery and compared with results in the literature. This study presents impressions from a 10-year period that exploration of the radial nerve is not necessary or mandatory to prevent radial nerve lesions for closed intramedullary nailing for HSFs in adults with a preoperative clinically normal radial nerve function.

The author declares not to have received any support from any organisation for the submitted work; has no financial relationships with any organisations that might have an interest in the submitted work, and has no other relationships or activities that could appear to have influenced the submitted work. Permission to use the presented data has been approved by the local ethical committee.

\section{Acknowledgement}

Dr. Michelle Fog Andersen for her role in assisting with gathering relevant patient data.

\section{REFERENCES}

1. Dilisio M, Nowinski R, Armodios M, Hatzidakis D, Fehringer E. Intramedullary nailing of the proximal humerus : evolution, technique, and results. J Shoulder Elbow Surg. $2016 ; 25(5): 30-8$.

2. Bumbasirević M, Lesić A, Bumbasirević V, Cobeljić G, Milosević I, Atkinson H. The management of humeral shaft fractures with associated radial nerve palsy : a review of 117 cases. Arch Orthop Trauma Surg. 2010 ; 130(4) : 519-22.

3. Claessen F, Peters R, Verbeek D, Helfet D, Ring D. Factors associated with radial nerve palsy after operative treatment of diaphyseal humeral shaft fractures. J Shoulder Elbow Surg. 2015 ; 24(11) : e307-11.

4. DeFranco M, Lawton J. Radial nerve injuries associated with humeral fractures. J Hand Surg Am. 2006; 31(4) : 655-63.

5. Nast-Kolb D. Changes and progress in fracture treatment of the humeral shaft. Orthopade. 1989 ; 18(3) : 208-13.

6. Huerta Lazcarro J, Luna Pizarro D. A comparison of the prevalence of radial nerve lesion after fixation of humeral shaft fractures with dynamic compression plate versus intramedullary nailing. Acta Ortop Mex. 2008 ; 22(5) : 28791.

7. Pollock F, Drake D, Bovill E, Day L. Treatment of radial neuropathy associated with fractures of the humerus. $J$ Bone Joint Surg Am. 1981 ; 63(2) : 239-43.

8. Shao Y, Harwood P, Grotz M, Limb D, Giannoudis P. Radial nerve palsy associated with fractures of the shaft of 
the humerus : a systematic review. J Bone Joint Surg Br. $2005 ; 87(12): 1647-52$.

9. Verga M, Peri Di Caprio A, Bocchiotti MA, Battistella F, Bruschi S, Petrolati M. Delayed treatment of persistent radial nerve paralysis associated with fractures of the middle third of humerus : review and evaluation of the long-term results of 52 cases. J Hand Surg Eur. 2007 ; S1753-1934.

10. Wang X, Zhang P, Zhou Y, Zhu C. Secondary radial nerve palsy after internal fixation of humeral shaft fractures. Eur $J$ Orthop Surg Traumatol. 2014 ; 24(3) : 331-3.

11. Grass G, Kabir K, Ohse J, Rangger C, Besch L, Mathiak G. Primary Exploration of Radial Nerve is Not Required for Radial Nerve Palsy while Treating Humerus Shaft Fractures with Unreamed Humerus Nails. Open Orthop J. $2011 ; 5: 319-23$.

12. Lin J. Locked nailing of spiral humeral fractures with or without radial nerve entrapment. Clin Orthop Relat Res. $2002 ; 403: 213-20$.

13. Wang J, Shen W, Chen W, Huang C, Shen Y, Chen T. Iatrogenic radial nerve palsy after operative management of humeral shaft fractures. $J$ Trauma. 2009 ; 66(3) : 800-3.
14. Bishop J, Ring D. Management of radial nerve palsy associated with humeral shaft fracture : a decision analysis model. J Hand Surg Am. 2009 ; 34(6) : 991-6.

15. AO Fracture Classification. The $A O$ (Arbeidsgemeinschaft für Osteosyntesefragen) Foundation, Switzerland.

16. Hoppenfeld S, deBoer P, Buckley R. Surgical Exposures in Orthopaedics : The Anatomic Approach, Fifth Edition. Wolters Kluwer ; 2016.

17. Lang N, Ostermann R, Arthold C, Joestl J, Platzer P. Retrospective case series with one year follow-up after radial nerve palsy associated with humeral fractures. Int Orthop. 2017 ; 41(1) : 191-196.

18. Böstman O, Bakalim G, Vainionpää S, Wilppula E, Pätiälä H, Rokkanen P. Radial palsy in shaft fracture of the humerus. Acta Orthop Scand. 1986 ; 57(4) : 316-9.

19. Karabay N, Toros T, Ademoğlu Y, Ada S. Ultrasonographic evaluation of the iatrogenic peripheral nerve injuries in upper extremity. Eur J Radiol. 2010 ; 73(2) : 234-40. 